hep-th/9801207

UCB-PTH-98/09

LBNL-41341

BU/HEP-98-04

UCSD-PTH-98/05

\title{
Gauge Theories with Tensors from Branes and Orientifolds
}

\author{
Csaba Csáki ${ }^{a}$, , Martin Schmaltz ${ }^{b}$, Witold Skiba $^{c}$, and \\ John Terning ${ }^{a}$ \\ ${ }^{a}$ Department of Physics, University of California, Berkeley, CA 94720 \\ and \\ Theory Group, Lawrence Berkeley National Laboratory, Berkeley, CA 94720 \\ csaki@thwk5.1bl.gov, terning@alvin.lbl.gov \\ ${ }^{b}$ Department of Physics, Boston University, Boston, MA 02215 \\ schmaltz@abel.bu.edu \\ ${ }^{c}$ Department of Physics, \\ University of California at San Diego, La Jolla CA 92093 \\ skiba@einstein.ucsd.edu
}

\begin{abstract}
We present brane constructions in Type IIA string theory for $\mathcal{N}=1$ supersymmetric $S O$ and $S p$ gauge theories with tensor representations using an orientifold 6-plane. One limit of these set-ups corresponds to $\mathcal{N}=2$ theories previously constructed by Landsteiner and Lopez, while a different limit yields $\mathcal{N}=1 S O$ or $S p$ theories with a massless tensor and no superpotential. For the $S p$-type orientifold projection comparison with the field theory moduli space leads us to postulate two new rules governing the stability of configurations of D-branes intersecting the orientifold. Lifting one of our configurations to M-theory by finding the corresponding curves, we re-derive the $\mathcal{N}=1$ dualities for $S O$ and $S p$ groups using semi-infinite D4 branes.
\end{abstract}

\footnotetext{
${ }^{1}$ Research Fellow, Miller Institute for Basic Research in Science.
} 


\section{Introduction}

A growing number of supersymmetric field theories have been realized as field theories on the world volume of various brane configurations. While brane dynamics provides information about field theory, one also learns about properties of brane configurations from field theory knowledge. One considers branes either in the context of perturbative string theory [1-14] or embedded in M-theory or F-theory [15-41].

Orientifold projection is a natural way of obtaining $S O$ and $S p$ theories on the world volume of branes, and it can also yield field theories with two-index tensors. Many results have been obtained by using an orientifold four plane [6, 17, 24, 25, 33, 42, 43]. In this paper we consider brane configurations with an orientifold projection realized by an orientifold six plane (O6-plane). These configurations allow us to describe gauge theories which have not been constructed previously using other brane configurations.

Our configurations can be understood as generalizations of the brane set-up considered by Landsteiner and Lopez [26] for $\mathcal{N}=2$ supersymmetric $S O$ and $S p$ theories. Landsteiner and Lopez (LL) considered D4 branes stretched between two parallel NS5 branes. In between the NS5 branes there are D6 branes and an O6-plane. The O6-plane amounts to a reflection involving the space-time directions which are orthogonal to the orientifold. As usual, the orientifold projection combines this spatial reflection with a parity inversion on the string world sheet.

In this paper, we break the $\mathcal{N}=2$ supersymmetry of the LL configuration by rotating the two NS5 branes. The rotation corresponds to adding a mass term for the adjoint chiral superfield in the field theory. A new interesting brane configuration is obtained when the two NS5 branes are rotated until they are parallel to the orientifold, then the corresponding field theory has an additional massless chiral superfield: a symmetric (antisymmetric) tensor in the case of $S O(S p)$. We investigate the Coulomb and Higgs branches for all our theories in detail and demonstrate agreement with the field theory expectations. For the $S p$ configuration we find that two new rules concerning the consistency of $\mathrm{D}$ branes and orientifolds are required. The "doubling rule" requires D branes which cross or are parallel to the orientifold to come in pairs (thus explaining why $S p$ theories only exist for even numbers of colors and flavors), and the "s'-rule" which is similar to the "s-rule" of Hanany and Witten [3], and which is necessary to reproduce the dimension of the Higgs branch of the field theory moduli space correctly.

Finally, we also consider brane configurations corresponding to $S O$ and $S p$ theories with only fundamental matter fields. We explicitly construct the corresponding M-theory curves and use them to re-derive Seiberg's duality for $S O$ and $S p$ gauge theories. This derivation is similar to the derivation of Seiberg's duality using an O4 plane in Ref. [24].

The paper is organized as follows. In the next section, we describe the basic brane configuration and determine the corresponding low-energy field theory. In Section 3, we study the Higgs branch of theories with flavors. In Section 4, we derive duality for $S O$ and 


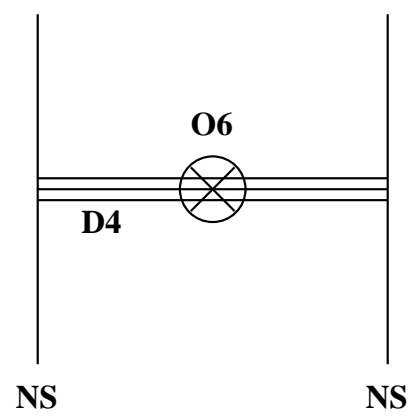

Figure 1: The brane configuration in Type IIA string theory giving rise to the pure $\mathcal{N}=2$ $S O$ or $S p$ theories. The $\otimes$ denotes the O6-plane which is perpendicular to the NS5 branes.

$S p$ with fundamentals in the context of string theory by considering brane motions. We discuss the embedding of this set-up in M-theory and present curves describing our brane configuration in Section 5. Finally, we conclude in the last section.

Certain elements of our paper have appeared very recently in Refs. [13, 14]. In particular, Brunner, Hanany, Karch and Lust [14 mention the brane configurations of Sec. 2. The counting of the $\mathcal{N}=2$ moduli spaces of Section 3 together with a slightly different statement of the s'-rule of Section 3 (with the same physical consequences) appeared in the work of Elitzur, Giveon, Kutasov and Tsabar [13].

\section{The Brane Set-up}

In this section we consider brane configurations in Type IIA string theory which give rise to $S O$ and $S p$ gauge groups. Our starting point is the $\mathcal{N}=2$ configuration of Ref. [26] presented in Fig. 1. In this configuration we have an O6-plane in the $\left(x_{0}, x_{1}, x_{2}, x_{3}, x_{7}, x_{8}, x_{9}\right)$ directions, which thus acts as a mirrorf in $\left(x_{4}, x_{5}, x_{6}\right)$. In addition, we have two NS5 branes in the $\left(x_{0}, x_{1}, x_{2}, x_{3}, x_{4}, x_{5}\right)$ directions which are mirror images of each other under the orientifold projection. There are also $N$ D 4 branes in the $\left(x_{0}, x_{1}, x_{2}, x_{3}, x_{6}\right)$ directions connecting the two NS5 branes. This theory, as discussed in Ref. [26], corresponds to $\mathcal{N}=2 S O(N)$ theory or $S p(N)$ theory depending on the orientifold charge. First we will discuss the $\mathcal{N}=1 S O(N)$ theory obtained from this $\mathcal{N}=2$ set-up by rotation of the NS5 branes. Then we repeat the discussion for $S p$ groups. The analysis for the $S p$ theories is very similar to the case of $S O$ groups, with the exception of an important subtlety which we discuss in detail. Throughout this paper, we use the complex coordinates $v=x_{4}+i x_{5}$ and $w=x_{8}+i x_{9}$. With this notation, in the $\mathcal{N}=2$ theory the NS5 branes are at a point in the $w$ plane and fill out the $v$ plane, while the $\mathrm{O} 6$ is at the origin of $v$ and fills out $w$.

\footnotetext{
${ }^{2}$ We present all of our brane configurations as embedded into the double cover of the orientifolded space.
} 


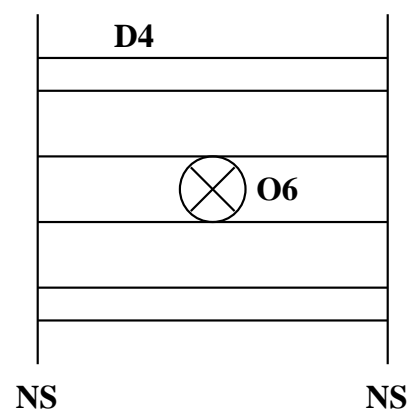

Figure 2: The moduli space of the $\mathcal{N}=2 S O(N)$ theory. Note that the $\mathrm{D} 4$ branes have to move in pairs away from the O6-plane.

\subsection{The $S O(N)$ Theories}

The $\mathcal{N}=2 S O(N)$ theory is given in Fig. 1. The moduli space of this theory corresponds to giving expectation values to the adjoint of the $S O(N)$ (which is an antisymmetric tensor). In the brane language this corresponds to sliding the D4 branes between the two parallel NS5 branes. At a generic point of the moduli space the adjoint VEV breaks $S O(N)$ to $U(1)^{r}$, where $r=N / 2$ for $N$ even and $r=(N-1) / 2$ for $N$ odd, which just corresponds to sliding all D4 branes apart from each other as illustrated in Fig. 2. Due to the O6 projection the D4 branes have to slide between the NS5 branes in pairs in the opposite directions, thus for $N$ even we get as a dimension of the moduli space $N / 2$. For $N$ odd one of the D4 branes is stuck at the O6, and the number of moduli is given by $(N-1) / 2$.

The $\mathcal{N}=2 S O(N)$ theory has an anomalous $U(1)_{R}$ symmetry, under which the adjoint field (the antisymmetric tensor of $S O(N)$ ) carries charge two. The $U(1)_{R}$ can be identified with rotations of the $v(45)$ plane, $v \rightarrow e^{i \theta} v$, we call this symmetry $R_{v}$. Thus, the $R_{v}$ charge of the adjoint is two.

Let us now rotate the NS5 branes slightly out of the $v$ plane into the $w$ plane (Fig. 3). Since the configuration must remain symmetric under the orientifold projection, both NS5 branes have to be rotated, but in opposite directions. As a result of the rotation, the D4 branes are fixed at the origin, they can not slide between the NS5 branes anymore. Thus the moduli space of the theory is lifted. This is exactly analogous to what happens in the $\mathcal{N}=2 S U(N)$ theories when one of the NS5 branes is rotated [18, 19].

In the field theory a mass for the adjoint chiral superfield is generated, breaking $\mathcal{N}=2$ to $\mathcal{N}=1$, and lifting the Coulomb branch of the theory. The antisymmetric tensor $A$ gets a small mass $W=\mu A^{2}$ when the two NS5 branes are slightly rotated. The $\mu \rightarrow 0$ limit corresponds to the $\mathcal{N}=2$ theory discussed before, and for generic $\mu$ we obtain pure $\mathcal{N}=1$ $S O(N)$ gauge theory with no massless chiral superfields. This theory has no moduli space.

However, we can observe that there is another limit of the rotated brane configuration 


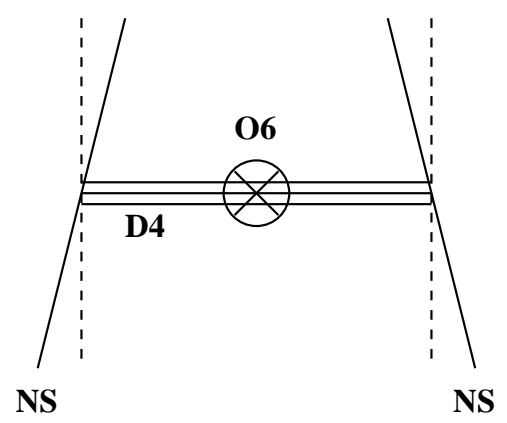

Figure 3: The brane configuration obtained by the rotation of the NS5 branes of the $\mathcal{N}=2$ $S O(N)$ theory.

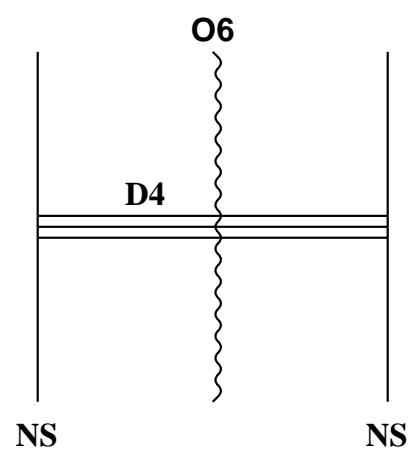

Figure 4: The brane configuration obtained in the $\mu \rightarrow \infty$ limit, which gives rise to $\mathcal{N}=1$ $S O(N)$ theory with a massless symmetric tensor, or $S p(N)$ with an antisymmetric tensor.

where the $S O(N)$ theory does have a moduli space. If the angle of rotation is $\pi / 2$ the two NS5 branes become parallel to each other and the O6-plane, and the D4 branes can slide between the NS5 branes again (Fig. (1). The emerging moduli space of the brane configuration must correspond to a field whose mass goes to zero in the field theory. We conjecture that this field transforms as a symmetric tensor under the $S O(N)$ gauge group. [. We now present four different arguments in support of this conjecture.

First we compare the moduli space of the field theory of $S O(N)$ with a symmetric tensor to that of the brane picture. In the brane picture the moduli space corresponds to the sliding of the D4 branes along the parallel NS5 branes (see Fig. 5). This obviously has $N$ moduli, and since a single D4 brane in the presence of an O6 corresponds to " $S O(1)$ ", this means that on a generic point of the moduli space the $S O(N)$ is completely broken. Let us now consider

\footnotetext{
${ }^{3}$ In the classical picture the trace of the symmetric tensor of $S O(N)$ appears as a modulus as well. This degree of freedom is presumably frozen by an infrared divergence of the quantum theory, similar to the trace of the adjoint of the $\mathcal{N}=2 S U(N)$ theories [16].
} 


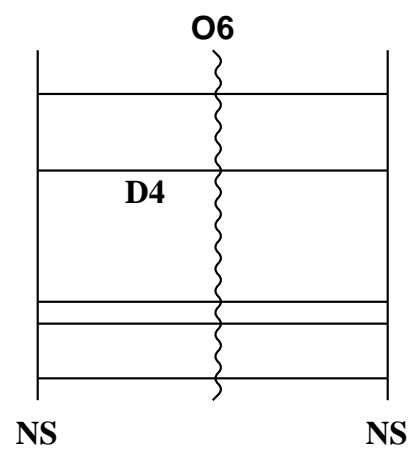

Figure 5: The moduli space of the $\mathcal{N}=1 S O(N)$ theory with a symmetric tensor. If all D4 branes move apart the gauge group is completely broken.

the unbroken group if we move away only one D4 brane (Fig. 6). Then we still have $N-1$ D4 branes on top of each other and thus this breaking corresponds to $S O(N) \rightarrow S O(N-1)$. This brane picture is in complete agreement with the field theory result. In the field theory, giving a $\mathrm{VEV}$

$$
V\left(\begin{array}{lllll}
1 & & & & \\
& 1 & & & \\
& & \ddots & & \\
& & & 1 & -(N-1)
\end{array}\right)
$$

to the symmetric tensor breaks the gauge group to $S O(N-1)$, and there is a massless symmetric tensor of the $S O(N-1)$ group remaining. This can be identified with the above brane motion where one of the D4 branes is sliding away from the remaining $N-1$. In general, the symmetric tensor $S$ of $S O(N)$ can completely break the $S O(N)$ group, which corresponds to moving all D4 branes apart from each other. Finally, the independent gauge invariant operators are given by $\operatorname{Tr} S, \operatorname{Tr} S^{2}, \ldots, \operatorname{Tr} S^{N}$ [4], and thus the number of moduli agrees with the brane prediction. In summary, we find complete agreement between the field theory and the brane moduli space. Note, that the antisymmetric tensor (adjoint) would not give the right dimension of moduli space, since an $\mathcal{N}=2 S O(2 M)$ theory has $M$ moduli.

As a second piece of evidence for establishing that the brane configuration of Fig. \& corresponds to $S O(N)$ with a symmetric tensor consider the brane set-up of Fig. 7 (we will discuss this brane configuration in more detail in Section 2.3). Here we have three NS5 branes, two of which are parallel to the O6-plane and are thus in the $\left(x_{0}, x_{1}, x_{2}, x_{3}, x_{8}, x_{9}\right)$ directions, while the third NS5 brane is at the same point as the O6 in the $x_{6}$ direction, but is perpendicular to it, since it is in the $\left(x_{0}, x_{1}, x_{2}, x_{3}, x_{4}, x_{5}\right)$ directions. This theory corresponds to $\mathcal{N}=1 U(N)$ theory with a symmetric tensor and its conjugate. We can however move the D4 branes away from the middle NS5 brane by sliding them between the parallel NS5 


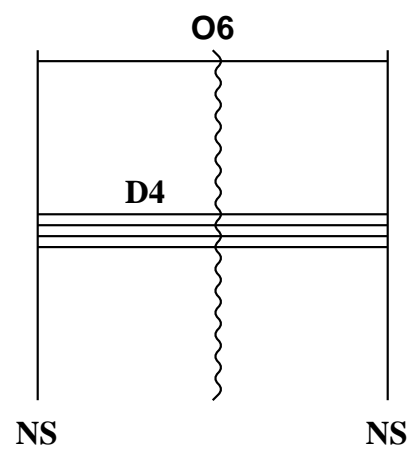

Figure 6: The brane configuration which corresponds to the breaking $S O(N) \rightarrow S O(N-1)$.

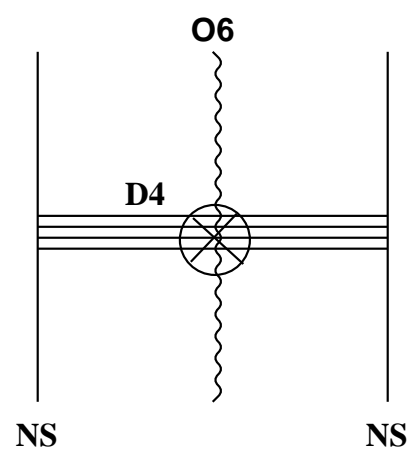

Figure 7: The brane configuration corresponding to an $\mathcal{N}=1 U(N)$ with a symmetric and a conjugate symmetric tensor. The $\otimes$ corresponds to a third NS5 brane perpendicular to the other two NS5 branes and the O6.

branes, and the resulting brane configuration is that of Fig. 国 (see Fig. 8). In the field theory this corresponds to the higgsing of $U(N)$ to $S O(N)$ by a VEV for a symmetric tensor which leaves one massless symmetric tensor transforming under the $S O(N)$ as expected from the brane picture.

As a third piece of evidence consider the argument in Section 2 of the work of Landsteiner and Lopez [26]. LL argue that $N$ D4 branes in an orientifold background give rise to two kinds of matter multiplets: an adjoint and a two-index tensor other than the adjoint. The boundary conditions imposed on the states on the D4 branes by the transverse NS5 branes project out some of these states. Which states are projected out depends on the orientation of the NS5 branes. If they are perpendicular to the $\mathrm{O} 6$ the tensor is projected out, thus leaving an $\mathcal{N}=2$ theory. If the NS5 branes are parallel to the O6 the adjoint and part of the tensor is projected out leaving $S O(N)$ with a symmetric tensor.

As final piece of evidence, consider the brane configuration of Fig. Q 9 . There, the theory 


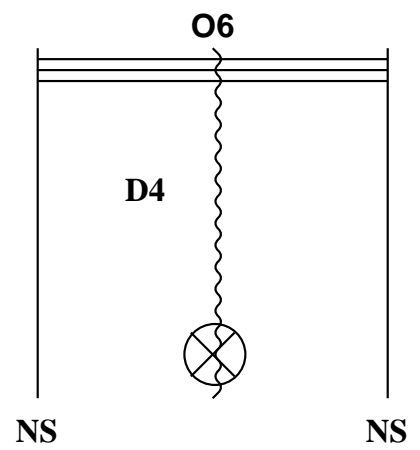

Figure 8: The brane configuration corresponding to breaking $U(N) \rightarrow S O(N)$ by giving an expectation value to the symmetric tensor.

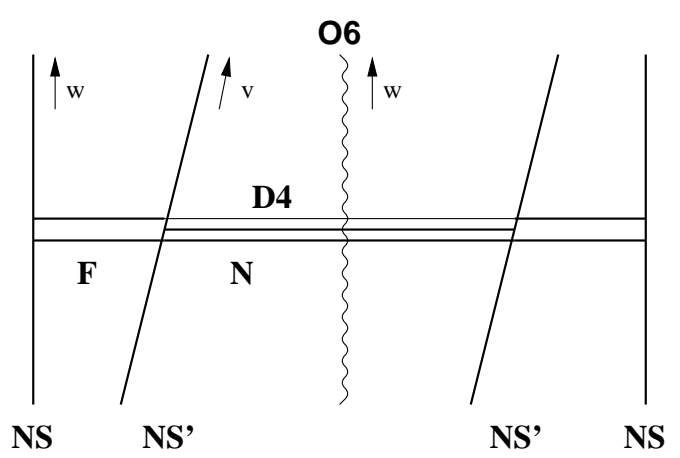

Figure 9: The brane configuration which corresponds to an $\mathcal{N}=2 S O(N)$ theory with $F$ flavors and with the $U(F)$ flavor symmetry gauged.

between the middle two NS5 branes is an $\mathcal{N}=2 S O(N)$ theory, and due to the additional $F$ D4 branes we get $F$ hypermultiplets of this $\mathcal{N}=2$ theory. Because the additional D4 branes end on two extra NS5 branes at the left and the right of the set-up, the $U(F)$ flavor symmetry is gauged. Thus, the field theory corresponding to Fig. 9 is given by

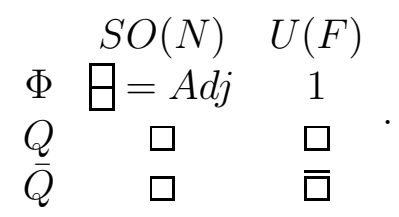

The superpotential of the theory is

$$
W=Q \Phi \bar{Q} .
$$

Now let us apply Seiberg's $\mathcal{N}=1$ duality to the $U(F)$ group (assuming that $F<N$ ). In the field theory this results in the theory 


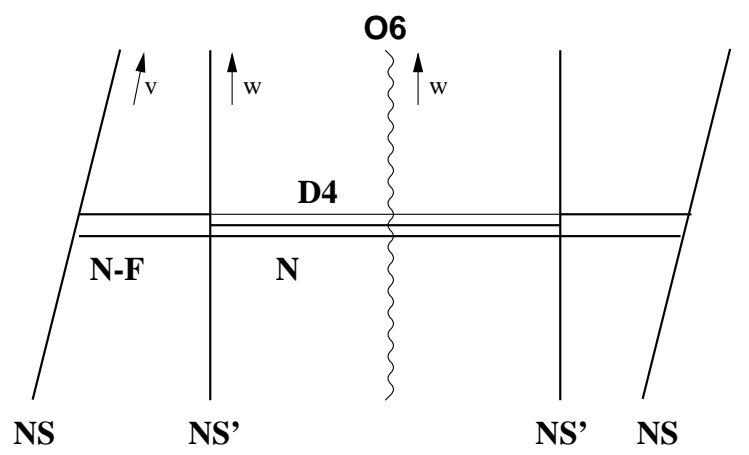

Figure 10: The brane configuration obtained by dualizing the $S U(F)$ group in Fig. 9 .

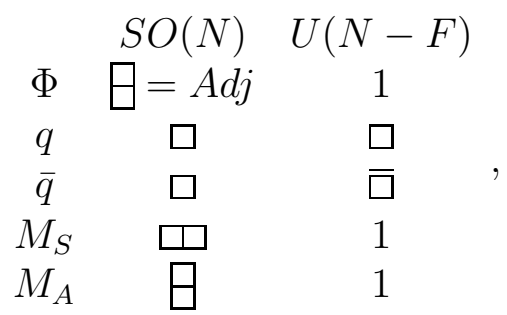

where $M_{A}$ is the antisymmetric part of the meson field, while $M_{S}$ is the symmetric part under the gauged $S O(N)$ subgroup of the original $U(N)$ flavor symmetry. The superpotential after the duality transformation is

$$
W=M_{A} \Phi+(q \bar{q})_{S} M_{S}+(q \bar{q})_{A} M_{A},
$$

which gives mass to the original adjoint $\Phi$ of $S O(N)$ together with the antisymmetric part of the meson $M_{A}$. Thus we are left with an $\mathcal{N}=1 S O(N)$ theory with a symmetric tensor and $F-N$ flavors ${ }^{\boxplus}$. The corresponding brane configuration is given in Fig. 10, from which one can see that the configuration of Fig. 1 must correspond to $S O(N)$ with a massless symmetric tensor.

Thus, we have established that the brane configuration of Fig. [4 corresponds to an $\mathcal{N}=1$ $S O(N)$ theory with a symmetric tensor. This theory has an anomalous $R$-symmetry under which the tensor has charge two. This symmetry can be identified with the rotations of the $w$ plane (89), $R_{w}$, and the $R_{w}$ charge of the tensor is two. In the limit of Fig. 1 which gives rise to the $S O(N)$ theory with a symmetric tensor, the mass of the adjoint is infinitely large, $\mu \rightarrow \infty$. Performing a small rotation around the $\frac{1}{\mu}=0$ limit we expect that the mass of

\footnotetext{
${ }^{4}$ Note that the additional $S O(N)$ singlet which is the trace of the symmetric tensor remains in the classical theory in this construction as well.
} 


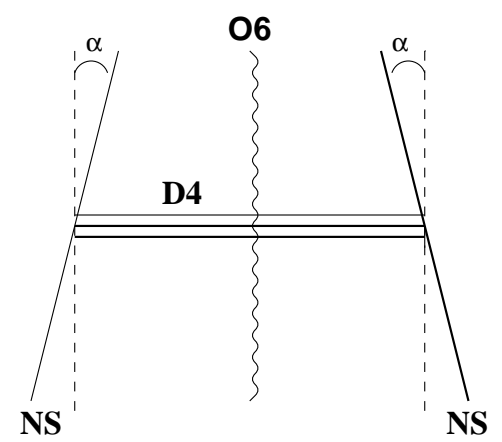

Figure 11: The general rotated brane configuration in Type IIA string theory giving rise to $\mathcal{N}=1 S O$ or $S p$ theories.

the symmetric tensor is proportional to $\frac{1}{\mu}$. This suggests that the configuration with the general rotation angle in Fig. 11 corresponds to $\mathcal{N}=1 S O(N)$ theory with an adjoint $A$ and a symmetric tensor $S$, and a superpotential

$$
W=\mu A^{2}+\frac{1}{\mu} S^{2}
$$

The anomalous R-symmetries of the fields are given by

$\begin{array}{ccc} & R_{v} & R_{w} \\ A & 2 & 0 \\ S & 0 & 2 \\ \mu & -2 & 2\end{array}$,

showing that the $R_{v}$ and $R_{w}$ charges of $\mu$ are consistent with our interpretation of $\mu$ as the adjoint mass and the inverse mass of the symmetric tensor.

\subsection{The $S p(2 N)$ Theories}

We now consider the same brane set-up as in the previous section except with negative O6 charge. The set-up of Fig. 1 gives rise to an $\mathcal{N}=2 S p(2 N)$ theory [26]. An important new element compared to the $S O(N)$ case is recognized by considering the fact that there is no $S p(N)$ group for $N$ odd. This leads us to postulate the

doubling rule: a single $D_{4}$ brane passing through the O6-plane is not invariant under an Sp-type $O 6$ projection, therefore the $D 4$ branes are necessarily paired.

The moduli space of the $\mathcal{N}=2 S p(2 N)$ theory is $N$ dimensional, corresponding to the breaking $S p(2 N) \rightarrow U(1)^{N}$ by the adjoint (which is the symmetric tensor) of $S p(2 N)$ (see Fig. 2). Again, the rotation of the NS5 branes of Fig. 1 gives mass $\mu S^{2}$ to the adjoint. 


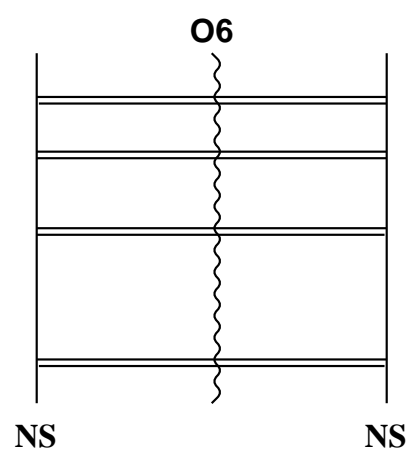

Figure 12: The moduli space of an $S p(2 N)$ theory with an antisymmetric tensor. Due to the orientifold projection the D4 branes can slide only in pairs between the NS5 branes.

The $\mu \rightarrow \infty$ limit ( $\pi / 2$ rotation) again gives a theory with a moduli space, corresponding to $\mathcal{N}=1 S p(2 N)$ theory with an antisymmetric tensor $A$. An important difference compared to $S O(N)$ appears when identifying the moduli space of the $S p(2 N)$ theory with an antisymmetric tensor. Due to the doubling rule the D4 branes must move in pairs.

A generic point on the moduli space is depicted in Fig. 12. Note that since two D4 branes are always on top of each other, the general unbroken subgroup is predicted to be $S U(2)^{N}$. In field theory the most general expectation value of the antisymmetric tensor is

$$
A=\left(\begin{array}{llll}
\omega_{1} & & & \\
& \omega_{2} & & \\
& & \ddots & \\
& & & \omega_{N}
\end{array}\right) \otimes i \tau_{2}
$$

which higgses $S p(2 N)$ to $S U(2)^{N}$, in agreement with the brane picture. Another way of seeing the agreement is to note that there are exactly $N$ independent gauge invariant operators of $S p(2 N)$ with an antisymmetric tensor $A$, given by $\operatorname{Tr} A J, \operatorname{Tr}(A J)^{2}, \ldots, \operatorname{Tr}(A J)^{N}$ where $J$ is the symplectic invariant 45 .

In all other respects the treatment of the $S p(2 N)$ theories is in complete analogy with the $S O(N)$ theories. The model obtained for a general rotation angle is $S p(2 N)$ with an adjoint (the symmetric tensor $S$ ) and an antisymmetric tensor $A$, with mass terms

$$
W=\mu S^{2}+\frac{1}{\mu} A^{2}
$$

where $\mu \rightarrow 0$ for the $\mathcal{N}=2$ theory, and $\mu \rightarrow \infty$ for the case when the NS5 branes and the O6-plane are parallel. The charges under the anomalous symmetries are: 


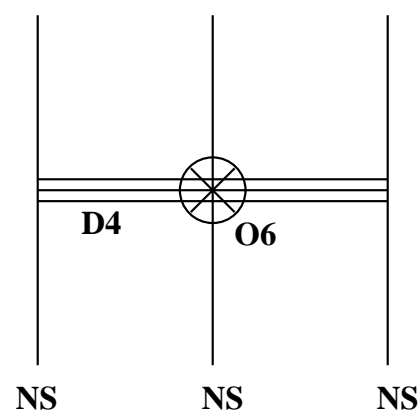

Figure 13: The brane configuration of Ref. 26] giving rise to $\mathcal{N}=2 U(N)$ theories with a symmetric or an antisymmetric flavor. The $\otimes$ denotes the O6-plane.

$\begin{array}{ccc} & R_{v} & R_{w} \\ S & 2 & 0 \\ A & 0 & 2 \\ \mu & -2 & 2\end{array}$.

\section{3 $U(N)$ Theories with Symmetric or Antisymmetric Tensors}

In this section we consider brane configurations corresponding to $\mathcal{N}=1 U(N)$ theories a symmetric flavor $(\square+\bar{\square})$ or an antisymmetric flavor $(\Theta+\bar{G})$. The starting point is the $\mathcal{N}=2 U(N)$ configuration of Landsteiner and Lopez [26] given in Fig. [13. The O6-plane is in the $\left(x_{0}, x_{1}, x_{2}, x_{3}, x_{7}, x_{8}, x_{9}\right)$ plane $(w)$, while the NS5 branes are in the $\left(x_{0}, x_{1}, x_{2}, x_{3}, x_{4}, x_{5}\right)$ $(v)$ plane. Depending on the charge of the O6-plane the theory is $\mathcal{N}=2 U(N)$ theory with a symmetric or an antisymmetric flavor. Rotation of the two outside NS5 branes gives a mass to the $U(N)$ adjoint breaking $\mathcal{N}=2$ to $\mathcal{N}=1$ (Fig. 14). The superpotential of the theory is

$$
W=\mu A^{2}+T A \bar{T},
$$

where $A$ is the $U(N)$ adjoint, and $T, \bar{T}$ are the symmetric or antisymmetric tensors of $U(N)$ and their conjugates. The second piece of the superpotential is the usual $\mathcal{N}=2$ superpotential term. Integrating out the massive adjoint field one can see that the configuration of Fig. 14 corresponds to an $\mathcal{N}=1 U(N)$ theory with a symmetric or antisymmetric flavor and a tree-level superpotential term

$$
W \propto \frac{1}{\mu}(T \bar{T})^{2} .
$$

\footnotetext{
${ }^{5}$ Quantum mechanically, the Abelian $U(1)$ factor of the $U(N)$ is not dynamical 16].
} 


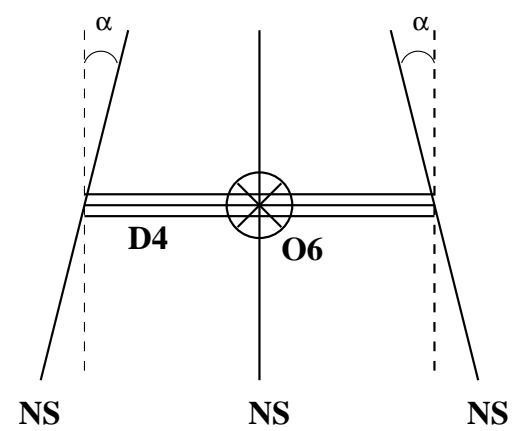

Figure 14: The brane configuration giving rise to $\mathcal{N}=1 U(N)$ theories with a symmetric or an antisymmetric flavor and a tree-level superpotential for them.

The configuration obtained in the limit when $\mu \rightarrow \infty$ corresponds to the $\mathcal{N}=1$ theory with $T, \bar{T}$ and no tree-level superpotential. This happens, when the two outside NS5 branes are parallel to each other, and to the O6-plane. The fact that the superpotential is vanishing in this limit can also be seen from the brane picture, where now the D4 branes can slide between the two outside NS5 branes, corresponding to expectation values of $T, \bar{T}$. This limiting case is depicted in Fig. 7, while the moduli space in this limit is illustrated in Fig. 8. This is the brane motion we have used to establish that the configuration of Fig. 1 corresponds to $\mathcal{N}=1 S O / S p$ theories with a tensor and no superpotential. Note that the moduli space of this $U(N)$ theory is exactly the same as the moduli space of the $\mathcal{N}=1 S O / S p$ theories with a massless tensor of Fig. 䍘, which agrees with the brane picture, since the presence of the middle NS5 brane is irrelevant for that.

Finally, we remark that the brane configuration corresponding to the $\mathcal{N}=1 U(N)$ theory with a massive symmetric or antisymmetric flavor is the one given in Fig. 15. The mass of the tensors corresponds to the separation of the D4 branes along the middle NS5 brane.

\section{The Higgs Branch}

In this section we are introducing $F$ D6 branes on each side of the O6-plane parallel to it. This will enable us to describe the $S O / S p$ theories with tensors and $F$ additional flavors. These theories, depending on the actual field content and superpotential usually have Coulomb branches, Higgs branches and mixed Coulomb-Higgs branches. We fill focus only on the pure Higgs branches of these theories, thus setting the tensor expectation value to zero. We are going to compare the dimension of the Higgs branch of the field theory when the gauge group is completely higgsed to the brane prediction. The moduli space appears in the brane picture because the D4 branes can break up between the D6 branes and the separate pieces can slide individually between the D6 branes. 


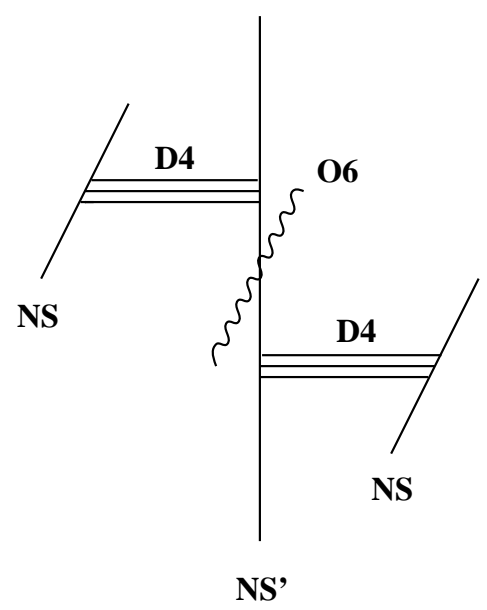

Figure 15: The brane configuration giving rise to $\mathcal{N}=1 U(N)$ theories with a massive symmetric or an antisymmetric flavor. The mass of the tensors corresponds to the separation of the D4 branes along the middle NS5 brane.

In order to count the dimension of the moduli space correctly, the following counting rules (as stated in Ref. [5]) have to be taken into account:

- a D4 brane between two D6 branes has two complex degrees of freedom (there are three real scalars corresponding to the three directions the D4 brane can move in and there is an additional scalar from the Kaluza-Klein compactification of the gauge field in the direction of the D4 brane).

- a D4 brane between a D6 brane and a parallel NS5 brane has one complex degree of freedom (there are two real scalar fields corresponding to the two directions the D4 brane can move in).

In addition to these counting rules, for a D4 brane stretched between an NS5 brane and a D6 brane perpendicular to the NS5 the following rule has to be obeyed [3]:

s-rule: there can be only one D4 brane suspended between a D6 brane and a perpendicular NS5 brane.

There are several pieces of evidence in favor of the s-rule. First of all, the neat fit with the field theory which we will show in the following subsections is only possible with the s-rule in place. Then, if there was more than one D4 brane stretched between the NS5 and the perpendicular D6 they would necessarily be on top of each other, a singular situation which presumably breaks supersymmetry [5]. Further evidence for the s-rule can be derived from M-theory. One can write down the M-theory curve for the given brane configuration and count the number of complex spheres which decouple from the curve and are free to slide along the D6 branes. This argument has been given in Ref. [18], the result is in exact agreement with the s-rule. 


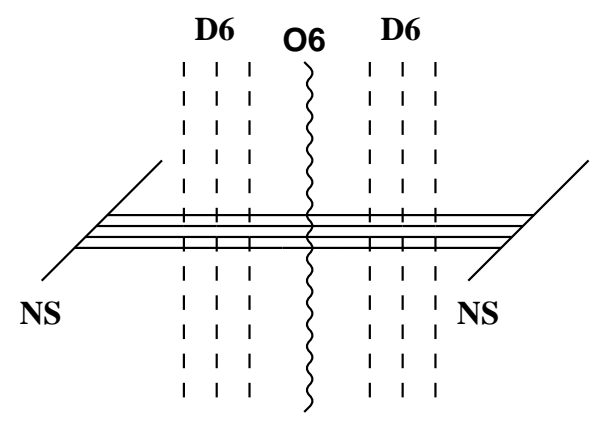

Figure 16: The $\mathcal{N}=2 S O(N)$ theory and $F$ hypermultiplets.

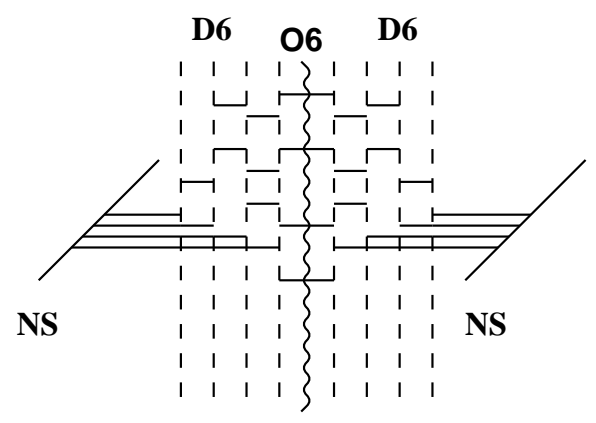

Figure 17: The moduli space of the Higgs branch of the $\mathcal{N}=2 S O(N)$ theory and $F$ hypermultiplets.

\subsection{The $S O(N)$ Theories}

Using the counting rules presented above we can check the dimension of the moduli space of the $S O$ theories of the previous section. First we consider the $\mathcal{N}=2 S O(N)$ theory.

The set-up of this theory is given in Fig. 16. The D6 branes fill out the $\left(x_{0}, x_{1}, x_{2}, x_{3}, x_{7}\right.$, $\left.x_{8}, x_{9}\right)$ directions just as the O6-plane, and are at definite points in $\left(x_{4}, x_{5}, x_{6}\right)$. The NS5 branes fill out the $\left(x_{0}, x_{1}, x_{2}, x_{3}, x_{4}, x_{5}\right)$ directions. If we want to identify the Higgs branch, we have to consider the case when the D6 branes are at the origin of $\left(x_{4}, x_{5}\right)$ (massless flavors). In this case, the D4 branes can break up and slide between the D6 branes. This will be the brane picture corresponding to the Higgs branch of the field theory. We want to find the maximal size of this Higgs branch. Taking into account the s-rule between the D6 and the NS5 branes one obtains for the Higgs branch a brane configuration as depicted in Fig. 17. 


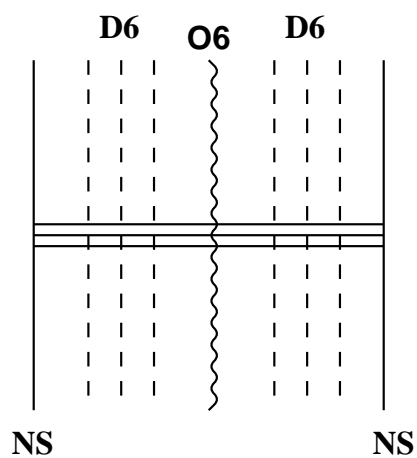

Figure 18: The basic set-up after including D6 branes parallel to the O6-plane. This will give rise to $S O / S p$ with a tensor and $F$ flavors.

Thus, the brane prediction for the size of the moduli space is

$$
\sum_{i=1}^{N} 2 i+(F-N) 2 N=2 F N-N^{2}+N .
$$

The field theory answer is exactly the same, which can be obtained by considering that we have $2 F N$ degrees of freedom from the quarks, but there are $N(N-1) / 2$ D-flatness conditions. However, due to the superpotential $Q A \bar{Q}$ of the $\mathcal{N}=2$ theory, where $Q, \bar{Q}$ are the $S O(N)$ vectors forming one hypermultiplet and $A$ is the $S O(N)$ adjoint, there are $N(N-1) / 2$ F-flatness conditions that these VEVs have to satisfy. Thus, the field theory result exactly matches the above brane prediction.

Let us now consider the other limiting case with a massless tensor, the $\mathcal{N}=1 S O(N)$ theory with a symmetric tensor and $F$ flavors. The brane set-up is given in Fig. 18. Now the NS5 and the D6 are parallel so the s-rule does not apply. Therefore the brane configuration for the Higgs branch is as in Fig. 19 .

Using these rules, the result for $S O(N)$ theories with $F$ flavors $(2 F$ fields in the vector representation) and a symmetric tensor is given by

$$
N+\underbrace{2 N+2 N+\ldots+2 N}_{F}=N+2 F N
$$

In the field theory, there are $2 F N$ complex degrees of freedom from the $2 F$ fields in the vector representation, $N(N+1) / 2$ from the symmetric tensor, but there are $N(N-1) / 2$ D-term conditions. Since there is no tree-level superpotential term present, the moduli space is

$$
2 F N+N(N+1) / 2-N(N-1) / 2=2 F N+N
$$

dimensional, again exactly in agreement with the brane prediction. 


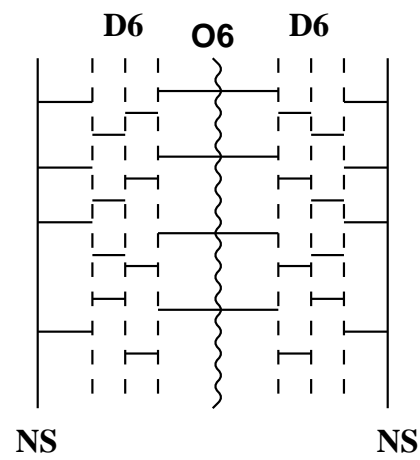

Figure 19: The moduli space of the Higgs branch of the $S O(N)$ theory with a symmetric tensor and $F$ massless flavors.

\subsection{The $S p(2 N)$ Theories}

The counting of the moduli space dimension for the $S p(2 N)$ theories is very similar to the case of $S O$ theories presented in the previous section, with the exception of two interesting subtleties arising from the negative projection of the orientifold. First, we have to take into account the doubling rule presented above (a single D4 brane passing through the O6-plane with negative charge is not invariant under the O6 projection) forcing the D4 branes which pass through the $\mathrm{O} 6$ to be paired. Furthermore, we find that in order to obtain the correct dimension of the moduli space the following generalized version of the s-rule has to hold:

s'-rule: when two or more D4 branes are forced to pass through a perpendicular D6 brane at the same point, only one of the D4 branes is allowed to break and end on the D6 brane.

In the original s-rule considered by Hanany and Witten the D4 branes were forced to be incident at a single point by their boundary condition on a perpendicular NS5 brane. In the case of a parallel NS5 brane the D4 branes are not forced to be coincident because they can be separated by sliding them between the parallel D6 and NS5 branes. Thus in that case the s'-rule does not apply. In the case at hand, the D4 branes are forced to come in pairs by the orientifold projection, and therefore only one of the two D4 branes can break on the D6 brane. Note that our s'-rule implies the counting rule given in Ref. [13]. The arguments in favor of the more general s'-rule rule are similar to those given for the usual s-rule. If more than one D4 brane were to end on the same D6 brane, then they would be necessarily on top of each other, which is a singular situation and is likely to break supersymmetry. We speculate that the s'-rule can also be derived by going to M-theory and counting the number of spheres which can decouple from the rest of the curve and slide along the sixbranes as done for the case of the s-rule in Ref. [18]. Finally, the neat fit between the field theory and string theory results presented below has to be considered a strong argument in favor of the s'-rule.

With this s'-rule in hand we can now calculate the size of the moduli space in the $S p$ 


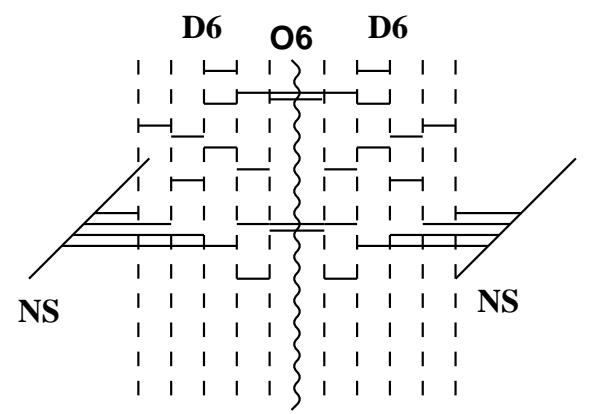

Figure 20: The moduli space of the Higgs branch of the $\mathcal{N}=2 S p(2 N)$ theory and $F$ hypermultiplets.

theories. First consider the $\mathcal{N}=2 S p(2 N)$ theories. The brane configuration respecting the s'-rule for the completely broken gauge group is given in Fig. 20. Thus the dimension of the moduli space for $S p(2 N)$ is predicted to be

$$
\sum_{i=1}^{2 N} 2 i+(F-2 N-2) 4 N+4 N=4 F N-4 N^{2}-2 N .
$$

In the field theory one has $4 F N$ degrees of freedom from the quarks, but there are $2 N(2 N+$ $1) / 2$ D-flatness conditions. Due to the superpotential term of $\mathcal{N}=2$ theories there are $2 N(2 N+1) / 2$ additional F-flatness conditions, exactly reproducing the brane prediction $4 F N-4 N^{2}-2 N$.

Next consider the $\mathcal{N}=1 S p(2 N)$ theory with an antisymmetric tensor. The most general brane configuration respecting the s'-rule is given in Fig. 21. The size of the moduli space for the $S p(2 N)$ theory is thus predicted to be

$$
2 N+\underbrace{4 N+4 N+\ldots+4 N}_{F-2}+2 N+2 N=6 N+4 N(F-2)=4 N F-2 N .
$$

The field theory result is in complete agreement. The $2 F$ quarks give rise to $2 F 2 N$ complex degrees of freedom, the antisymmetric tensor to $2 N(2 N-1) / 2$, but there are $2 N(2 N+1) / 2$ D-flatness conditions, resulting in $4 N F+N(2 N-1)-N(2 N-1)=4 N F-2 N$ degrees of freedom. Thus, we conclude that the counting of moduli space is correctly reproduced in every example once the s'-rule is taken into account. 


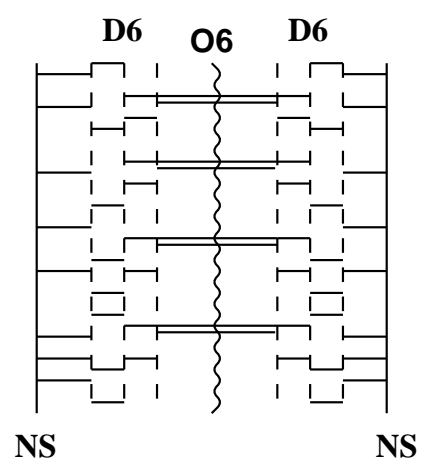

Figure 21: The moduli space of the Higgs branch of the $S p(N)$ theory with an antisymmetric tensor and $F$ massless flavors.

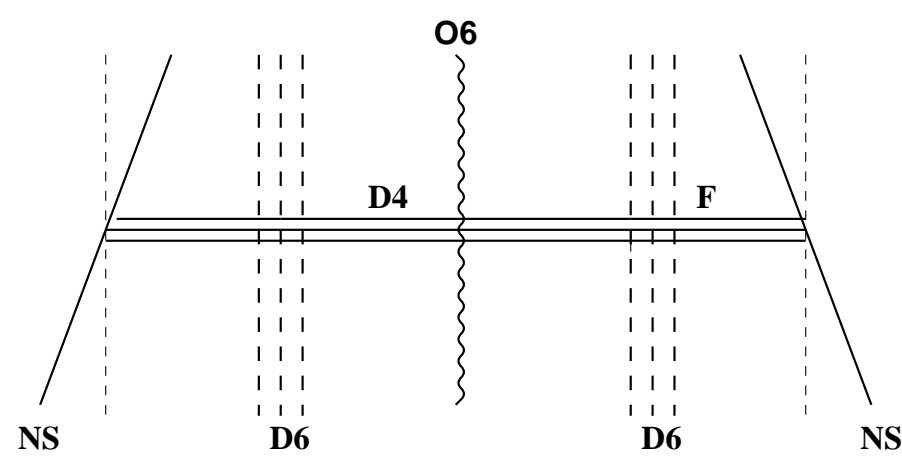

Figure 22: The brane set-up of the electric $S O / S p$ theory with $F$ flavors.

\section{$4 \mathcal{N}=1$ Duality from String Theory}

\subsection{Duality of $\mathcal{N}=1 S O$ and $S p$ theories}

Using the brane configurations presented in the previous sections, one can re-derive the $\mathcal{N}=1$ dualities for $S O$ and $S p$ groups of Refs. [46, 47, 48]. In the context of string theory these dualities have been obtained first in Ref. [6] with the use of an orientifold four plane.

In order to obtain an $\mathcal{N}=1 S O$ or $S p$ theory without tensors, we consider the general case of Fig. 11 with $\alpha$ not equal to 0 or $\pi / 2$. In this case we have an $\mathcal{N}=1$ theory with a massive adjoint and a massive tensor. In order to obtain the flavors of $S O$ and $S p$ we have to introduce $F$ additional D6 branes on both sides of the O6-plane parallel to it (Fig. 22). The resulting theory is $\mathcal{N}=1 S O$ or $S p$ with $F$ flavors ( $2 F$ vectors of $S O$ or $2 F$ fundamentals of $S p)$. However, the presence of the massive tensors induces a tree-level superpotential term for the flavors. This can be seen by considering the $\mathcal{N}=2$ configuration of Fig. 18. This 


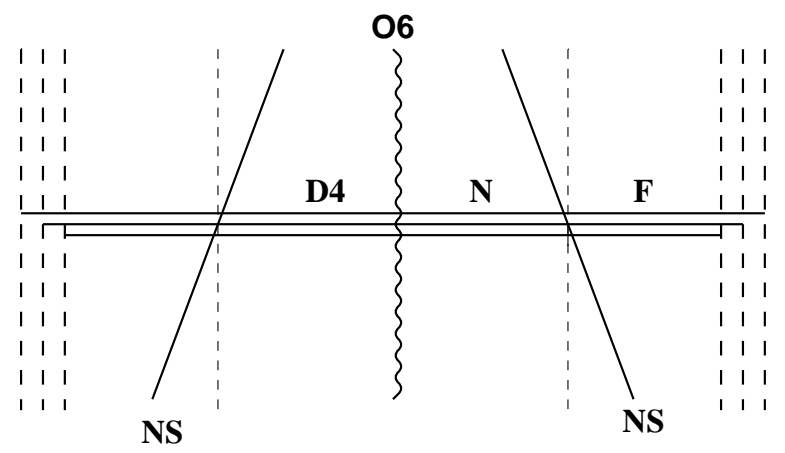

Figure 23: The brane set-up obtained after moving the D6 branes through the NS5 branes.

theory has a tree-level superpotential of the form

$$
W_{\mathcal{N}=2}=Q A \bar{Q}
$$

where $A$ is the adjoint of $S O / S p$ while the $Q, \bar{Q}$ are the flavors forming $\mathcal{N}=2$ hypermultiplets. Rotating the two NS5 branes generates a mass for the adjoint (the massive tensor appears as well, but since it does not couple to the flavors its presence does not leave an imprint on the moduli space, and we will ignore it in this section). Thus the full superpotential of the $\mathcal{N}=1$ theory is

$$
W=Q A \bar{Q}+\mu A^{2}
$$

integrating out the massive adjoint we get a superpotential

$$
W \sim(Q \bar{Q})^{2} .
$$

One can easily check that the counting of the dimension of the moduli space from the brane picture agrees with the field theory result. The simplest way to see this is to realize that the counting of the moduli space in the brane picture as well as in the field theory is exactly the same as for the $\mathcal{N}=2$ theories discussed in the previous section.

In order to obtain the dual description, we will move the D6 and the NS5 branes through each other, and use the linking number argument of Ref. [3] to obtain the number of D4 branes created. The linking numbers for the NS5 branes are given by

$$
L_{5}=\frac{1}{2}\left(n_{6 L}-n_{6 R}\right)+n_{4 R}-n_{4 L}
$$

where $n_{6 L, R}$ are the D6 branes to the left or right of the NS5 branes, and similarly $n_{4 R, L}$ are the D4 branes to the right or left of the NS5 branes. The linking numbers of the D6 branes are

$$
L_{6}=\frac{1}{2}\left(n_{5 L}-n_{5 R}\right)+n_{4 R}-n_{4 L}
$$




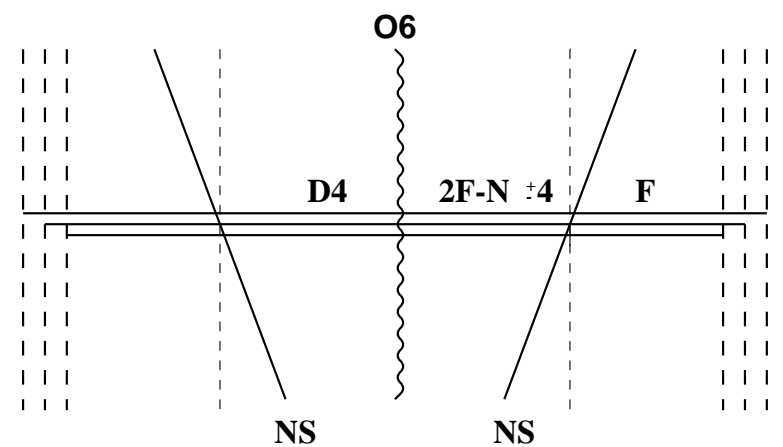

Figure 24: The brane set-up obtained after moving the NS5 branes through each other and the 06.

where $n_{5 L, R}$ are the NS5 branes to the left or right of the D6 brane, while $n_{4 R, L}$ are the D4 branes to the right or left of the D6 brane. We assume that for the linking number argument the O6-plane can be treated as \pm 4 D6 branes.

First, we move the D6 branes on the right all the way to the right (and their mirrors all the way to the left) as shown in Fig. 23. Considering the linking number $L_{6}$ of a given D6 brane, we can see that that there has to be one additional D4 brane ending on it after the move to conserve $L_{6}$. This is because originally $L_{6}=-\frac{1}{2}$, while after the move it is $L_{6}^{\prime}=\frac{1}{2}-n_{4 L}$, thus $n_{4 L}$ has to be one. There is one additional D4 brane created for every D6 brane, altogether there are $F$ D4 branes created on each side of the O6.

Next, consider moving the NS5 branes through each other and through the O6-plane. The resulting set-up for the magnetic theory is depicted in Fig. 24. To determine the number of D4 branes stretched between the two NS5 branes we consider conservation of $L_{5}$ linking number. The linking number of the NS brane on the left is originally $L_{5}=-\frac{1}{2}( \pm 4)+N-F$, since there are $N$ D 4 branes on the right, $F$ D 4 branes on the left and \pm 4 D 6 branes which correspond to the orientifold on the right. In the final state of Fig. 24 there are \pm 4 D6 branes on the left of the NS brane, $F \mathrm{D} 4$ branes on the right and $\tilde{N} \mathrm{D} 4$ branes on the left, thus $L_{5}^{\prime}=\frac{1}{2}( \pm 4)+F-\tilde{N}$, from which we obtain $\tilde{N}=2 F-N \pm 4$ which is the correct size of the dual gauge group. Furthermore, we can move the D6 branes through the NS5 branes back again, resulting in the configuration of Fig. 25. This is just like the configuration of the electric theory with a different gauge group, which does correspond to $S O(2 F-N+4)$ or $S p(2 F-N-4)$ with $F$ flavors and a superpotential

$$
W \sim(q \bar{q})^{2},
$$

where $q, \bar{q}$ are the dual quarks of $S O(2 F-N+4) / S p(2 F-N-4)$. This is in agreement with the field theory result, since from the $\mathcal{N}=1$ duality we expect to get the dual gauge group $S O(2 F-N+4)$ or $S p(2 F-N-4)$ and a superpotential term

$$
W_{\text {dual }}=M^{2}+M q \bar{q},
$$




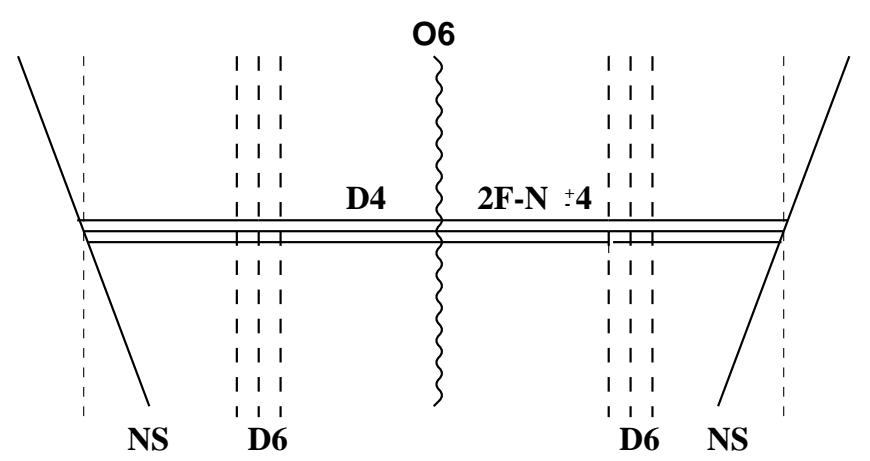

Figure 25: The brane set-up obtained after moving the D6 branes back through the NS5 branes.

where the first term comes from the $(Q \bar{Q})^{2}$ tree-level superpotential of the electric theory, while the $M q \bar{q}$ term is the additional superpotential required for duality. Thus the mesons are massive and one can integrate them out, leaving the superpotential

$$
W_{\text {dual }} \sim(q \bar{q})^{2}
$$

This is exactly what we find in the brane picture of Fig. 25.

\subsection{Duality of $U(N)$ with a Symmetric or Antisymmetric Flavor}

Let us present one more example of duality, which takes advantage of another brane configuration described previously. In Section 2.3 we outlined a configuration that gives a $U(N)$ theory with a flavor of symmetric (antisymmetric) tensors. To that configuration we add $F$ D6 branes as illustrated in Fig. 26. These additional six branes provide $F$ flavors in the fundamental representation. As we already described in Section 2.3 this theory has a tree-level superpotential which includes the term $(T \bar{T})^{2}$, where $T$ is a symmetric or an antisymmetric tensor depending on the charge of the orientifold plane. This is the theory considered in Sections 3 and 4 of Ref. [49], where duality was demonstrated after addition of the superpotential term $W \sim(T \bar{T})^{k+1}$. The field theory corresponding to our brane configuration has additional superpotential terms which act as perturbations. We discuss the (important) effect of these superpotential terms in the field theory at the end of this section.

Having already dealt with duality in a very similar set-up we can immediately derive the dual for this example. We keep the D6 branes fixed and move only the outer NS5 branes. First, we move them closer to the orientifold, passing them through the D6 branes. Then, we move the NS5 branes across each other and the O6 and the NS5 brane in the center, and finally move them outside the D6 branes. This final configuration is illustrated in Fig. 27. The number of D4 branes created by this motion is identical to the one for $S O(S p)$ duality with fundamental flavors. For example, this can be seen by determining the linking numbers 


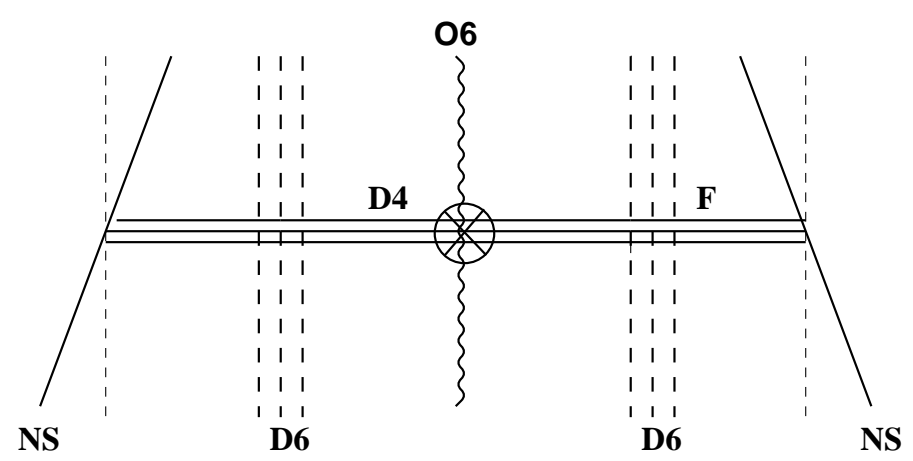

Figure 26: The brane configuration corresponding to the electric $S U(N)$ theory with a symmetric or an antisymmetric flavor and $F$ fundamental flavors. The $\otimes$ denotes an NS5 brane perpendicular to the O6.

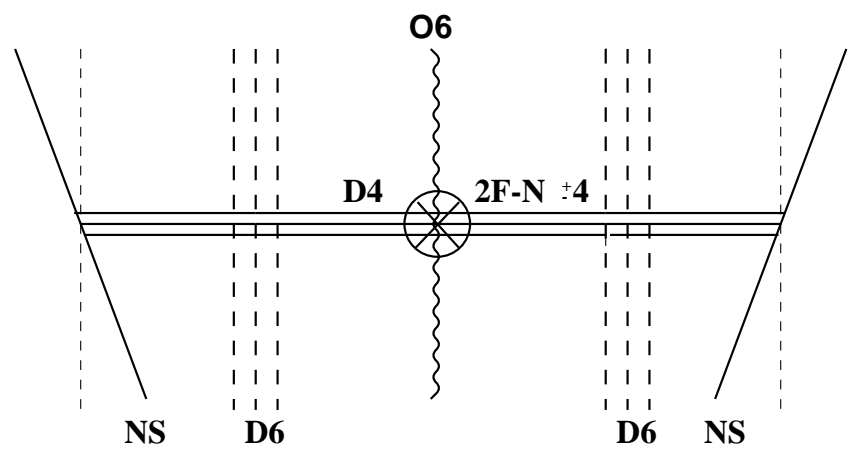

Figure 27: The magnetic configuration obtained after moving the NS5 branes through the D6 branes and each other

of the various branes before and after the moves. Thus after the move there are $2 F-N \pm 4$ D4 branes stretching between the NS branes. The configuration is similar to our starting set-up, except for a different number of D4 branes; it describes a theory with $S U(2 F-N \pm 4)$ gauge group with a superpotential of the same form as the one in the electric theory.

This result requires an explanation. The electric theory was constructed by rotating the outer two NS5 branes of the $\mathcal{N}=2$ theory, that is by giving a mass to the adjoint superfield. The resulting $\mathcal{N}=1$ theory with a massive adjoint has a superpotential

$$
W=\mu A^{2}+T A \bar{T}+Q A \bar{Q},
$$

and after integrating out the adjoint, the superpotential is

$$
W \sim(T \bar{T})^{2}+(Q \bar{Q})^{2}+Q \bar{T} T \bar{Q} .
$$

The dual which we read off of our brane construction is thus an $S U(2 F-N \pm 4)$ gauge 
theory with $F$ flavors, a symmetric (or antisymmetric) tensor flavor, and the superpotential

$$
W \sim(\tilde{\tilde{t}})^{2}+(\tilde{q} \overline{\tilde{q}})^{2}+\tilde{q} \tilde{\tilde{t}} \tilde{t} \tilde{q} .
$$

We now proceed to compare this result with the expected field theory answer. The field theory result can be obtained by using the dual presented in Ref. [49] for the theory with the superpotential $W=(T \bar{T})^{2}$ and adding the two terms $(Q \bar{Q})^{2}+Q \bar{T} \bar{T}$ as perturbations. The chiral ring of the theory without perturbation consists of two meson operators $M_{0}=Q \bar{Q}$, $M_{1}=Q \bar{T} T \bar{Q}$ and two tensors of the flavor group $P=Q \bar{T} Q, \bar{P}=\bar{Q} T \bar{Q}$. The dual is an $S U(3 F-N \pm 4)$ gauge theory with fundamental meson fields $P, \bar{P}, M_{0}, M_{1}$, dual quarks $q, \bar{q}$, dual tensors $t, \bar{t}$ and the superpotential

$$
W \sim(t \bar{t})^{2}+M_{1} q \bar{q}+M_{0} q \bar{t} t \bar{q}+P q \bar{t} q+\bar{P} \bar{q} t \bar{q}+M_{0}^{2}+M_{1}
$$

The last two terms correspond to the perturbations present in our brane set up. We see that the meson field $M_{0}$ is massive and should be integrated out, leading to a $(q \bar{t} t \bar{q})^{2}$ term in the superpotential. Furthermore, the linear term $M_{1}$ forces VEVs for the dual quarks $q$, $\bar{q}$. Since $M_{1}$ is an $F$ by $F$ matrix in flavor space, all dual quarks get VEVs. They break the magnetic gauge group $S U(3 F-N \pm 4)$ to $S U(2 F-N \pm 4)$. The dual quarks are eaten by the Higgs mechanism, while the tensors decompose into tensors of $S U(2 F-N \pm 4), F$

flavors and singlets. The singlets get masses with the $P$ and $\bar{P}$ fields from the $P q \bar{t} q+\bar{P} \bar{q} t \bar{q}$ terms. The remaining two terms in the superpotential yield $W=(\tilde{\tilde{t}})^{2}+(\tilde{q} \overline{\tilde{q}})^{2}+\tilde{q} \tilde{t} \tilde{t} \tilde{q}$, where these are fields coming from the decomposition of the tensor fields $t$ and $\bar{t}$. This is the same superpotential and gauge group which we obtained from the brane move.

\section{M-theory}

\section{$5.1 S O(2 N)$}

We now proceed to construct new M-theory curves for the $S O(2 N)$ theory. (The extension of all these results to $S O(2 N+1)$ is trivial.) The construction includes an O6-plane which is at a point in $v$ and extends in the $w$ direction and $x_{9}$. There are two NS5 branes, one on each side (in $x_{6}$ ) of the O6-plane (they are mirror images) extending in the $w_{ \pm}$directions where

$$
w_{ \pm}=w \pm \mu v
$$

The NS5 branes have 2N D4 branes stretched between them, and $F$ semi-infinite D4 branes extending to the left (negative $x_{6}$ direction) from the left NS5 brane, and mirrors of these on the right. This set-up is summarized in Fig. 28, and it describes an $S O(2 N)$ theory with $F$ flavors, that is $2 F$ vectors. To describe the orientifold we note that it carries 


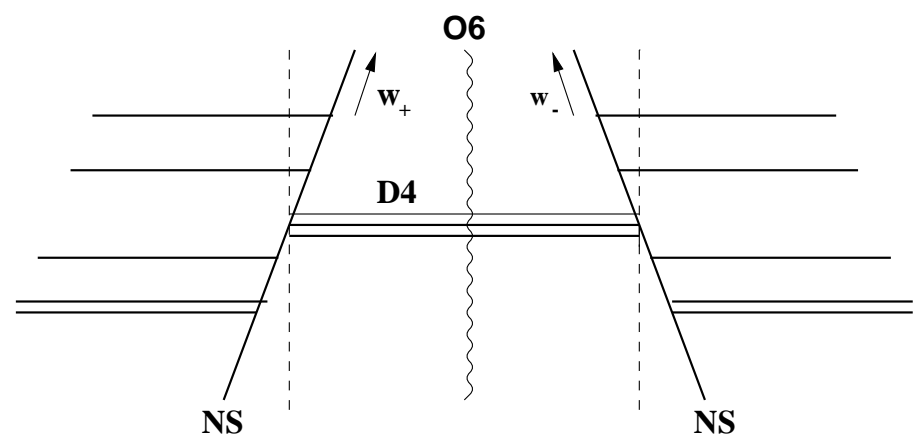

Figure 28: The brane set-up corresponding to $\mathcal{N}=1 S O / S p$ theories with massive flavors from semi-infinite D4 branes.

D6 brane charge four, and assume that its effects can be described in the same fashion as four coincident D6 branes. A D6 brane of type IIA string theory is described by Taub-NUT space in $\mathrm{M}$ theory. For our purposes it is sufficient to use one of the complex structures of this space and then the orientifold is given by [26]:

$$
x y=v^{4},
$$

where $y \sim t=\exp (-s / R)$ for large $y$ and $x \sim t^{-1}$ for large $x$. Here $s=x_{6}+i x_{10}$. To describe the curve in this space we need two equations in the complex variables $v, w, x$ and $y$. We will simply present the curves, and then give evidence that they are correct:

$$
w_{+} w_{-}=\mu \xi
$$

and

$$
(\mu \xi)^{N-1} y \prod_{i=1}^{F}\left(\frac{w_{+}-m_{i}}{w_{-}-m_{i}}\right)=v^{2} w_{+}^{2 N-2}
$$

where

$$
\xi=\left(\Lambda_{N, F}^{3(N-1)-F} \prod_{i}^{F} m_{i}\right)^{\frac{1}{N-1}}
$$

It is straightforward to check that equations (5.2), (5.3), and (5.4) are invariant under the orientifold projection which exchanges:

$$
\begin{aligned}
x & \leftrightarrow y \\
v & \leftrightarrow-v \\
w & \leftrightarrow w
\end{aligned}
$$




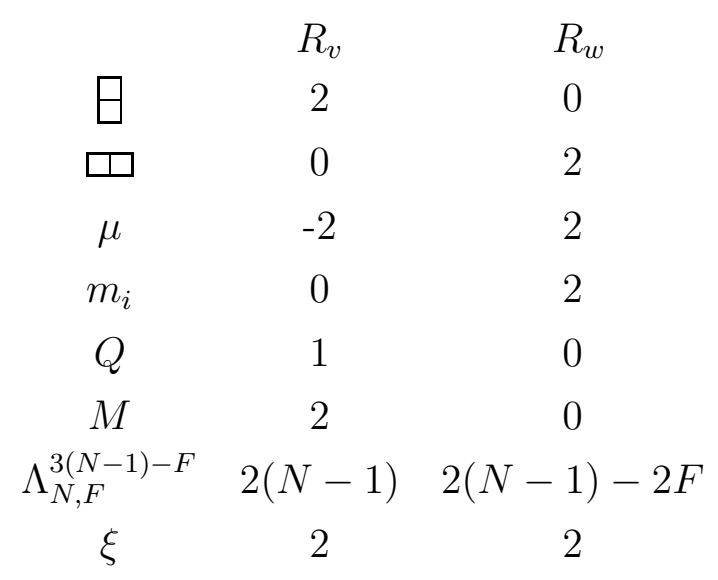

Table 1: R-charges for $S O(2 N)$.

which imply $w_{+} \leftrightarrow w_{-}$. Furthermore the curve is invariant under $R_{v}$ and $R_{w}$ as defined in Table 1.

The most convincing evidence that the curves are correct is seen by taking the string theory limit, as we describe in detail in the next subsection. The string theory limit is achieved by taking $R \rightarrow 0$, where $R$ is the radius of the compact 11-th dimension. Taking this limit we see that the M-theory curve reduces to the string theory picture of 5 branes and 4 branes described above. Readers who are already convinced that the curves are correct may skip ahead.

\section{$5.2 \quad$ The String Theory Limit}

We now consider the string theory limit of the curve. We take $R \rightarrow 0$ with

$$
\Lambda_{N, F}^{3(N-1)-F} \sim e^{-L / R}
$$

while holding $L$ and $m_{i}$ fixed. In order to see what the $\mathrm{M}$ theory configuration reduces to we first consider a point on the curve with fixed large $w_{+} \gg m_{i}$, and search for solutions with large $y \sim t=e^{-s / R}$ (here $s<0$ ). Equation (5.3) implies that

$$
w_{-} \rightarrow 0
$$

while equation (5.4) implies that

$$
e^{-L / R} e^{-s / R} \sim \text { const. } w_{+}^{2(N-1)-F},
$$

so $s=-L$ in the limit $R \rightarrow 0$. These solutions correspond to the NS5 brane on the left. Next consider

$$
w_{+} \sim m_{i}+e^{-c / R} .
$$


Equation (5.4) implies that

$$
e^{-L / R} e^{-s / R} e^{-c / R} \sim \text { const. }
$$

so $s=-L-c$. Thus we see $F$ solutions (one for each $m_{i}$ ) extending to the left (negative $x_{6}$ ) that approach $w_{+}=m_{i}$ as $s \rightarrow-\infty$. These are precisely the $F$ semi-infinite $\mathrm{D} 4$ branes on the left. The mirror solutions can be found on the right for large $x$ (instead of large $y$ ).

Finally consider

$$
w_{+} \sim e^{-c / R}
$$

Equation (5.4) implies that for $2 c<L /(N-1)$

$$
e^{-L / R} e^{-s / R} \sim \text { const. } e^{-2 N c / R}
$$

so $s=-L+2 N c$ and these solutions sit to the right of the NS5 brane and are the color D4 branes.

In M-theory the NS5 branes can bend in the $x_{6}$ direction, corresponding to the running coupling of the field theory. We can also check that our curve correctly reproduces this behavior. Taking a slice of the brane-surface with large, fixed $y$ we should find that the number of solutions of the curve corresponds precisely to the number of branes in string picture. To see this we rewrite equation (5.4) in terms of $y$ and $w_{+}$. Using equation (5.3) and

$$
v=\frac{1}{2 \mu}\left(w_{+}-\frac{\mu \xi}{w_{+}}\right)
$$

we find:

$$
(\mu \xi)^{N-1} y \prod_{i=1}^{F}\left(w_{+}-m_{i}\right) w_{+}^{F-2 N+4}=\left(w_{+}^{2}-\mu \xi\right)^{2} \prod_{i=1}^{F}\left(\mu \xi-m_{i} w_{+}\right)
$$

There are three cases: a) $F \geq 2 N$, there are $2 F-2 N+4$ solutions, $F$ with $w_{+} \approx m_{i}$ (the "flavor-branes") and $F-2 N+4$ with $w_{+} \approx 0$ (this corresponds to the NS5 brane on the right bending to the left); b) $2 N-4<F<2 N$, there are $F+4$ solutions, $F$ with $w_{+} \approx m_{i}$, $2 N-F$ with large $w_{+}$(the NS5 brane on the left bends to the left), and $F-2 N+4$ solutions with $w_{+} \approx 0$ (these solutions are related to the O6-plane, and in the string theory sit to the right of the NS5 brane on the left as describe above); c) $F \leq 2 N-4$, there are $2 N$ solutions, $F$ with $w_{+} \approx m_{i}$, and $2 N-F$ with large $w_{+}$(again the NS5 brane on the left bends to the left). 


\subsection{Decoupling a Flavor}

It is also a simple exercise to check that decoupling a flavor works correctly. For example take $m_{F} \rightarrow \infty$, this has the effect of replacing

$$
\prod_{i=1}^{F} \rightarrow \prod_{i=1}^{F-1}
$$

and making the identification (from one-loop matching of the gauge coupling)

$$
\Lambda_{N, F}^{3(N-1)-F} \prod_{i}^{F} m_{i}=\Lambda_{N, F-1}^{3(N-1)-(F-1)} \prod_{i}^{F-1} m_{i}
$$

\subsection{Duality}

We also see that the M-theory curve correctly describes the dual gauge theory by taking the string theory limit $(R \rightarrow 0)$ with $\Lambda_{N, F} \rightarrow \infty$ (for the asymptotically free case) rather than $\Lambda_{N, F} \rightarrow 0$ as we did above. Equation (5.7) then implies that $L<0$. The intrinsic scale, $\tilde{\Lambda}_{\tilde{N}, F}$, of the dual theory is related to $\Lambda_{N, F}$ by:

$$
\Lambda_{N, F}^{b} \tilde{\Lambda}_{\tilde{N}, F}^{\tilde{b}}=\mu^{b+\tilde{b}}
$$

where $b$ and $\tilde{b}$ are the one-loop $\beta$-function coefficients, and $\mu$ is a scale which is approximately equal to the string scale by dimensional analysis. Thus, we see that the $\operatorname{limit} \Lambda_{N, F} \rightarrow \infty$ corresponds to $\tilde{\Lambda}_{\tilde{N}, F} \rightarrow 0$. To make things simpler we rewrite equation (5.4) with $w_{-}$ expressed in terms of $w_{+}$and vice versa by use of Equation (5.3). More explicitly:

$$
(\mu \xi)^{N-1} y \prod_{i=1}^{F}\left(\frac{\frac{\mu \xi}{w_{-}}-m_{i}}{\frac{\mu \xi}{w_{+}}-m_{i}}\right)=v^{2}\left(\frac{\mu \xi}{w_{-}}\right)^{2 N-2}
$$

is equivalent to

$$
(\mu \xi)^{\tilde{N}-1} y \prod_{i=1}^{F}\left(\frac{w_{-}-\mu M_{i}}{w_{+}-\mu M_{i}}\right)=v^{2} w_{-}^{2 \tilde{N}-2}
$$

where

$$
\tilde{N}=F-N+2
$$

\footnotetext{
${ }^{6}$ We are stating this for the case of asymptotically free electric and magnetic theories $(b, \tilde{b}>0)$, the extension to the other cases $(b \leq 0$ or $\tilde{b} \leq 0)$ is straightforward and gives further evidence for duality. For details see Ref. [23].
} 
corresponding to the dual gauge group $S O(\tilde{N})$, and $M_{i}$ is the meson VEV:

$$
M_{i}=\frac{\xi}{m_{i}}
$$

Note that

$$
\xi=\left(\tilde{\Lambda}_{\tilde{N}, F}^{3(\tilde{N}-1)-F} \prod_{i}^{F} m_{i}\right)^{\frac{1}{N-1}}
$$

In order to hold the meson VEV fixed in the $R \rightarrow 0$ limit we must take:

$$
m_{i}^{F-N+1} \sim e^{L / R} \rightarrow 0
$$

Thus we see that the string theory limit of the M-theory curve with $\Lambda_{N, F} \rightarrow \infty$ (i.e. $L<0$ ) gives precisely the string theory picture of the dual gauge theory.

\section{$5.5 S p(2 N)$}

Next we consider the M-theory curves for the $S p(2 N)$ theory. The construction again includes an O6-plane (but with the opposite charge) at a point in $v$ and extending in the $w$ direction and $x_{9}$. There are two NS5 branes, one on each side (in $x_{6}$ ) of the O6-plane extending in the $w_{ \pm}$directions. The NS5 branes have $2 N$ D4 branes stretched between them, and $F$ semi-infinite D4 branes extending to the left from the left NS5 brane, and mirrors of these on the right (Fig. 28). Again we need three equations in the complex variables $v, w, x$ and $y$. As before we give the curves and then the evidence that they are correct. The O6-plane is described by [26]:

$$
x y=v^{-4} .
$$

We also have

$$
w_{+} w_{-}=\mu \xi
$$

and

$$
(\mu \xi)^{N+1} y \prod_{i=1}^{F}\left(\frac{w_{+}-m_{i}}{w_{-}-m_{i}}\right)=v^{-2} w_{+}^{2 N+2}
$$

It is straightforward to check that equations (5.25), (5.26), and (5.27) satisfy the correct symmetries and to check that decoupling a flavor works correctly. 


\subsection{The String Limit}

We now consider the string theory limit $R \rightarrow 0$ of the curve with

$$
\xi^{N+1} \sim e^{-L / R},
$$

$L>0$ and $m_{i}$ fixed. We first consider fixed $w_{+} \gg m_{i}$, and search for solutions with large $y \sim t=e^{-s / R}$. Equation (5.26) implies that

$$
w_{-} \rightarrow 0
$$

while equation (5.27) implies that

$$
e^{-L / R} e^{-s / R} \sim \text { const. } w_{+}^{2 N-F},
$$

so $s=-L$ in the limit $R \rightarrow 0$. These solutions correspond to the NS5 brane on the left. Next consider

$$
w_{+} \sim m_{i}+e^{-c / R} .
$$

Equation (5.4) implies that

$$
e^{-L / R} e^{-s / R} e^{-c / R} \sim \text { const. },
$$

so $s=-L-c$. Thus we see $F$ solutions (one for each $m_{i}$ ) extending to the left that approach $w_{+}=m_{i}$ as $s \rightarrow-\infty$. These are the $F$ semi-infinite D4 branes on the left. The mirror solutions can be found on the right for large $x$. Finally consider

$$
w_{+} \sim e^{-c / R},
$$

equation (5.27) implies that for $2 c<L /(N+1)$

$$
e^{-L / R} e^{-s / R} \sim \text { const. } e^{-(2 N+2) c / R},
$$

so $s=-L+(2 N+2) c$ and these solutions sit to the right of the NS5 brane; they are the color D4 branes.

We can also check the curve by counting the number of solutions for large, fixed $y$ and comparing to the number of branes in the string picture. Using equations (5.14) and (5.26) we rewrite equation (5.27) in terms of $y$ and $w_{+}$:

$$
(\mu \xi)^{N+1} y \prod_{i=1}^{F}\left(w_{+}-m_{i}\right) w_{+}^{F-2 N-4}\left(w_{+}^{2}-\mu \xi\right)^{2}=\prod_{i=1}^{F}\left(\mu \xi-m_{i} w_{+}\right)
$$

There are again three cases: a) $F \geq 2 N+4$, there are $2 F-2 N$ solutions, $F$ with $w_{+} \approx m_{i}$ (the "flavor-branes"), $F-2 N-4$ with $w_{+} \approx 0$ (this corresponds to the NS5 brane on the right bending to the left) and four solutions with $w_{+}^{2} \approx \mu \xi$ (associated with the O6-plane); b) $2 N+2 \leq F<2 N+4$, there are $F+4$ solutions, $F$ with $w_{+} \approx m_{i}$, and four solutions with $w_{+}^{2} \approx \mu \xi$ (associated with the O6-plane); c) $F<2 N+2$, there are $2 N+6$ solutions, $F$ with $w_{+} \approx m_{i}$, and $2 N+2-F$ with large $w_{+}$(the NS5 brane on the left bends to the left), and four solutions with $w_{+}^{2} \approx \mu \xi$ (associated with the O6-plane). 


\subsection{Duality}

We can also see that the M-theory curve correctly describes the dual gauge theory by simply rewriting Equation (5.27) and taking the limit $R \rightarrow 0$ with $\Lambda \rightarrow \infty$. The curve,

$$
(\mu \xi)^{N+1} y \prod_{i=1}^{F}\left(\frac{\frac{\mu \xi}{w_{-}}-m_{i}}{\frac{\mu \xi}{w_{+}}-m_{i}}\right)=v^{-2}\left(\frac{\mu \xi}{w_{-}}\right)^{2 N+2}
$$

is equivalent to

$$
(\mu \xi)^{\tilde{N}+1} y \prod_{i=1}^{F}\left(\frac{w_{-}-\mu M_{i}}{w_{+}-\mu M_{i}}\right)=v^{-2} w_{-}^{2 \tilde{N}+2}
$$

where

$$
\tilde{N}=F-N-2
$$

corresponding to the dual gauge group $S p(2 \tilde{N})$. Again we see that this correctly reproduces dual gauge theory in the string theory limit.

\section{Conclusions}

We have investigated brane realizations of $\mathcal{N}=1 S O$ and $S p$ gauge theories with two-index tensor representations. Our construction employed an orientifold six plane. By studying various limiting cases we have established the field content and the tree-level superpotential of our brane construction. The important limiting cases correspond to $\mathcal{N}=2$ theory with tensor hypermultiplets and $\mathcal{N}=1$ theories with massless tensors and no superpotential. For these cases, we have checked that the brane degrees of freedom are in exact correspondence with the flat directions in field theory.

Consistency requirements impose two new restrictions on brane configurations involving an orientifold six plane. First, orientifolds with the $S p$-type projection can be only crossed by pairs of D4 branes. A single D4 brane is not invariant under the projection of $S p$-type. Second, the s-rule needs to be generalized to our s'-rule in the presence of an orientifold six plane with negative charge. Our first observation says that D4 branes crossing an O6-plane have to group into pairs invariant under the orientifold projection. Then, according to the s'-rule only one of the two branes in each such pair can end on a given D6 brane parallel to an O6-plane.

We have also studied duality for $S O$ and $S p$ groups both in the string theory framework and in M-theory. In string theory, we included D6 branes which transform under flavor symmetries. The dual gauge group and meson excitation are identified when the original brane configuration is deformed by moving NS5 and D6 branes. In M-theory, we included 
semi-infinite branes and found the equations describing the single brane configuration. This configuration encodes the information about the dual gauge group and the vacuum expectation values of the meson fields.

\section{Acknowledgments}

We are grateful to Jan de Boer, Kentaro Hori, Hirosi Ooguri, Yaron Oz, Erich Poppitz and Raman Sundrum for useful discussions. We also thank Jan de Boer, Yaron Oz and Zheng Yin for comments on the manuscript. C.C. is a research fellow of the Miller Institute for Basic Research in Science. C.C. and J.T. are supported in part by the National Science Foundation under grant PHY-95-14797, and are also partially supported by the Department of Energy under contract DE-AC03-76SF00098. M.S. is supported by the U.S. Department of Energy under grant \#DE-FG02-91ER40676. W.S. is supported by the Department of Energy under contract DE-FG03-97ER405046.

\section{References}

[1] J. Polchinski, S. Chaudhuri and C. Johnson, hep-th/9602052; J. Polchinski, hepth/9611050.

[2] A. Strominger, Phys. Lett. 383B, 44 (1996), hep-th/9512059; P. Townsend, Phys. Lett. 373B, 68 (1996), hep-th/9512062.

[3] A. Hanany and E. Witten, Nucl. Phys. B492, 152 (1997), hep-th/9611230.

[4] J. de Boer, K. Hori, H. Ooguri, Y. Oz and Z. Yin, Nucl. Phys. B493, 148 (1997), hep-th/9612131.

[5] S. Elitzur, A. Giveon and D. Kutasov, Phys. Lett. 400B, 269 (1997), hep-th/9702014; S. Elitzur, A. Giveon, D. Kutasov and E. Rabinovici, Nucl. Phys. B505, 202 (1997), hep-th/9704104.

[6] N. Evans, C. Johnson and A. Shapere, Nucl. Phys. B505, 251 (1997), hep-th/9703210.

[7] J. Barbon, Phys. Lett. 402B, 59 (1997), hep-th/9703051.

[8] J. Brodie and A. Hanany, Nucl. Phys. B506, 157 (1997), hep-th/9704043.

[9] O. Aharony and A. Hanany, Nucl. Phys. B504, 239 (1997), hep-th/9704170.

[10] A. Brandhuber, J. Sonnenschein, S. Theisen and S. Yankielowicz, Nucl. Phys. B502, 125 (1997), hep-th/9704044; 
[11] A. Hanany and A. Zaffaroni, Nucl. Phys. B509, 145 (1998), hep-th/9706047; hepth/9801134.

[12] K. Landsteiner, E. Lopez and D.A. Lowe, hep-th/9801002.

[13] S. Elitzur, A. Giveon, D. Kutasov and D. Tsabar, hep-th/9801020.

[14] I. Brunner, A. Hanany, A. Karch and D. Lust, hep-th/9801017.

[15] A. Klemm, W. Lerche, P. Mayr. C. Vafa and N. Warner, Nucl. Phys. B477, 746 (1996), hep-th/9604034.

[16] E. Witten, Nucl. Phys. B500, 3 (1997), hep-th/9703166.

[17] K. Landsteiner, E. Lopez and D. A. Lowe, Nucl. Phys. B507, 197 (1997), hepth/9705199; A. Brandhuber, J. Sonnenschein, S. Theisen and S. Yankielowicz, Nucl. Phys. B504, 175 (1997), hep-th/9705232.

[18] K. Hori, H. Ooguri and Y. Oz, hep-th/9706082.

[19] E. Witten, Nucl. Phys. B507, 658 (1997), hep-th/9706109.

[20] A. Brandhuber, N. Itzhaki, V. Kaplunovsky, J. Sonnenschein and S. Yankielowicz, Phys. Lett. 410B, 27 (1997), hep-th/9706127.

[21] A. Hanany, M. Strassler and A. Zaffaroni, hep-th/9707244.

[22] J. de Boer and Y. Oz, hep-th/9708044; J. de Boer, K. Hori, H. Ooguri and Y. Oz, hep-th/9711143.

[23] M. Schmaltz and R. Sundrum, hep-th/9708015.

[24] C. Csáki and W. Skiba, Phys. Lett. 415B, 31 (1997), hep-th/9708082.

[25] J. de Boer, K. Hori, H. Ooguri and Y. Oz, hep-th/9801060.

[26] K. Landsteiner and E. Lopez, hep-th/9708118.

[27] E. Witten, hep-th/9712028.

[28] J. Lykken, E. Poppitz and S. Trivedi, hep-th/9708134; hep-th/9712193.

[29] A. Giveon and O. Pelc, hep-th/9708168.

[30] J. Erlich, A. Naqvi and L. Randall, hep-th/9801108. 
[31] N. Evans and M. Schwetz, hep-th/9708122; N. Evans, hep-th/9801159.

[32] T. Nakatsu, K. Ohta, T. Yokono and Y. Yoshida, hep-th/9707258.

[33] C. Ahn, K. Oh and R. Tatar, hep-th/9707027; hep-th/9708127; Phys. Lett. 416B, 75 (1998), hep-th/9709096; hep-th/9712005.

[34] S. Terashima, hep-th/9712172.

[35] J. Furukawa, hep-th/9706121.

[36] S. Nam, K. Oh and S. Sin, hep-th/9707247; S. Sin, hep-th/9801032.

[37] A. Fayyazuddin and M. Spalinski, Nucl. Phys. B508, 219 (1997), hep-th/9706087; hepth/9711083.

[38] P. Howe, N. Lambert and P. West, hep-th/9710034; N. Lambert and P. West, hepth/9712040.

[39] S. Katz and C. Vafa, Nucl. Phys. B497, 146 (1997), hep-th/9606086; S. Katz, A. Klemm and C. Vafa, Nucl. Phys. B497, 173 (1997), hep-th/9609239; S. Katz and C. Vafa, Nucl. Phys. B497, 196 (1997), hep-th/9611090; M. Bershadsky, A. Johansen, T. Pantev, V. Sadov and C. Vafa, Nucl. Phys. B505, 153 (1997), hep-th/9612052.

[40] H. Ooguri and C. Vafa, Nucl. Phys. B500, 62 (1997), hep-th/9702180; C. Vafa and B. Zwiebach, Nucl. Phys. B506, 143 (1997), hep-th/9701015; S. Katz, P. Mayr and C. Vafa, hep-th/9706110.

[41] C. Ahn and K. Oh, Phys. Lett. 412B, 274 (1997), hep-th/9704061; R. Tatar, hepth/9704198; C. Ahn, hep-th/9705004; C. Ahn and R. Tatar, Phys. Lett. 413B, 293 (1997), hep-th/9705106.

[42] E. Gimon and J. Polchinski, Phys. Rev. D54, 1667 (1996), hep-th/9601038.

[43] J. Blum and K. Intriligator, Nucl. Phys. B506, 223 (1997), hep-th/9705030.

[44] G. Dotti and A. Manohar, hep-th/9712010.

[45] P. Cho and P. Kraus, Phys. Rev. D54, 7640 (1996), hep-th/9607200; C. Csáki, W. Skiba and M. Schmaltz, Nucl. Phys. B487, 128 (1997), hep-th/9607210.

[46] N. Seiberg, Nucl. Phys. B435, 129 (1995), hep-th/9411149.

[47] K. Intriligator and N. Seiberg, Nucl. Phys. B444, 125 (1995), hep-th/9503179. 
[48] K. Intriligator and P. Pouliot, Phys. Lett. 353B, 471 (1995), hep-th/9505006.

[49] K. Intriligator, R. Leigh and M. Strassler, Nucl. Phys. B456, 567 (1995), hepth/9506148. 